\title{
Third-trimester Cerebroplacental Ratio as a Predictor of Neonatal Outcome in a Low-risk Pregnancy
}

\author{
Nandita Maitra ${ }^{1}$, Pragati Prajapati ${ }^{2}$, Tosha M Sheth ${ }^{3}$, Palak Vaishnav ${ }^{4}$, Purvi K Patel ${ }^{5}$
}

\begin{abstract}
Objective: To study the role of the cerebroplacental ratio (CPR) in the prediction of adverse neonatal outcomes and mode of delivery in low-risk women at 37-41 weeks' gestation.

Materials and methods: Three hundred and seventy low-risk women with singleton pregnancy at 37-41 weeks of gestation, with known last menstrual period (LMP), were prospectively evaluated over a period of 1 year in a medical college setting. Doppler examination was performed and the indices recorded. Defined maternal and neonatal outcomes were studied. Nonparametric tests such as test for comparison of population means, test for comparison of proportions, and tests of diagnostic accuracy were performed. Receiver operating characteristic (ROC) curves were used to determine the area under the curve.

Results: Total 78 subjects had CPR below 5 th centile. The mean CPR value at the 5 th centile was 1.2. The mean (SD) of CPR for the total population was 1.48 (+0.39); the mean (SD) value for CPR was lowest for the infants requiring neonatal intensive care unit (NICU) admission (1.12 \pm 0.22 ). The CPR below the 5th centile had an independent association with APGAR $<7$ at 5 minutes, induced labor, and NICU admission. Birth weight centile did not affect this association.

Conclusion: The CPR below the 5th centile was associated with a higher risk of obstetric intervention for intrapartum fetal compromise and adverse perinatal outcomes in subjects with low-risk pregnancy at term.

Keywords: Adverse, Cerebroplacental, Neonatal, Outcome, Ratio, Term.

Journal of South Asian Federation of Obstetrics and Gynaecology (2020): 10.5005/jp-journals-10006-1781
\end{abstract}

\section{INTRODUCTION}

The cerebroplacental ratio (CPR) is emerging as an important predictor of adverse fetal outcome. ${ }^{1}$ This ratio quantifies the redistribution of cardiac output by dividing the Doppler indices of the middle cerebral artery (MCA) with that of the umbilical artery (UA). The cerebroplacental index ratio is the ratio of the MCA pulsatility index (PI) to the UA PI. Redistribution of cerebral blood flow or the brain sparing effect can be indicated by low CPR. The brain sparing effect is seen when circulatory adaptation occurs with chronic hypoxia in the form of cerebral vasodilatation to preserve blood flow to the brain.

Because the CPR reflects both the placental status and fetal response, it has been reported as being a more sensitive Doppler index for predicting perinatal outcome. ${ }^{2-4}$ The CPR is also an earlier predictor of adverse outcome than the biophysical profile, UA, or MCA alone.

Most studies on the clinical use of CPR have focused on assessment of small for gestational age (SGA) fetuses. There are few studies on the role of CPR in low-risk women. About $63 \%$ cases of intrapartum hypoxia occur in pregnancies with no antenatal risk factors, making it difficult to identify the fetus at risk of complications in labor. ${ }^{5,6}$ One study reported that the prediction of adverse outcome by low CPR was better if the time interval between assessment and delivery was $\leq 2$ weeks rather than $>2$ weeks and suggested that the performance of screening by CPR at 36 weeks may be superior to that at 32 weeks. ${ }^{7}$

Prior et al.' ${ }^{1}$ prospectively evaluated 400 appropriate for gestational age (AGA) fetuses at term and reported an abnormal CPR in $11 \%$. Of those who underwent cesarean delivery (CD) for fetal distress, $36.4 \%$ had an abnormal CPR compared with $10.1 \%$ $(p<0.001)$ who had a normal CPR. ${ }^{1}$
${ }^{1-5}$ Department of Obstetrics and Gynecology, Medical College Baroda,
Vadodara, Gujarat, India

Corresponding Author: Nandita Maitra, Department of Obstetrics and Gynecology, Medical College Baroda, Vadodara, Gujarat, India, Phone: +919824357844, e-mail:n.maitra03@gmail.com

How to cite this article: Maitra N, Prajapati P, Sheth TM, et al. Thirdtrimester Cerebroplacental Ratio as a Predictor of Neonatal Outcome in a Low-risk Pregnancy. J South Asian Feder Obst Gynae 2020;12(3): 150-154.

Source of support: Nil

Conflict of interest: None

This prospective observational study was undertaken to study the following objective: the role of CPR in the prediction of adverse neonatal outcomes and the mode of delivery in low-risk women at 37-41 weeks' gestation.

\section{Materials and Methods}

Three hundred and seventy low-risk women with singleton pregnancy at 37-41 weeks of gestation, with confirmed gestational age, were prospectively evaluated over a period of 1 year.

\section{Study Design}

Prospective cross-sectional study

\section{Sample Size Calculation}

The exclusion criteria were as follows:

- Multiple pregnancy

- Previously identified fetal growth restriction

() The Author(s). 2020 Open Access This article is distributed under the terms of the Creative Commons Attribution 4.0 International License (https://creativecommons. org/licenses/by-nc/4.0/), which permits unrestricted use, distribution, and non-commercial reproduction in any medium, provided you give appropriate credit to the original author(s) and the source, provide a link to the Creative Commons license, and indicate if changes were made. The Creative Commons Public Domain Dedication waiver (http://creativecommons.org/publicdomain/zero/1.0/) applies to the data made available in this article, unless otherwise stated. 
- Known fetal anomaly

- Women with medical disorder other than preeclampsia

- Preeclampsia

- Previous one or two cesarean section

- Women with previous uterine surgery such as myomectomy

Maternal age, parity, booking blood pressure, weeks of gestation, body mass index (BMI), history of medical disorder, previous history of still birth, or neonatal death were documented. Fetal biometry, amniotic fluid index, and PI of UA and MCA were recorded.

Doppler examinations were performed by two examiners using a 3.5-MHz convex probe (Esaote My Lab 50) with spatial peak temporal average intensities below $50 \mathrm{~mW} / \mathrm{cm}^{2}$ and the highpass filter at $50-100 \mathrm{~Hz}$. All measurements were performed in the absence of a gross fetal body or breathing movements. For each examination, the mean value of three consecutive waveforms was recorded. The angle of insonation was kept below $30^{\circ}$.

The following variables were recorded:

- UA PI obtained from a free loop of the umbilical cord;

- MCA PI measured in the straight portion of the artery avoiding head compression by the transducer; and

- CPR derived as a simple ratio of the MCA PI divided by the UA PI.

- Analysis was performed using a CPR percentile of $<5$ th centile. A cut-off value of less than 5 th centile has been taken recognizing that 0.6765 CPR MoM equates to the 5th centile. The CPR percentile was calculated from the calculator available on http:// medicinafetalbarcelona.org/calc/. ${ }^{8}$

- Birth weight centile was calculated from the calculator available at http://www.gestation.net/cc/10/624762.htm. ${ }^{9}$

Women found to have abnormal CPR values were followed up with weekly assessment and induction of labor was offered to the women when fetal compromise was suspected. Labor was managed as per local protocols and guidelines. After delivery, intrapartum and neonatal outcome data were recorded. The following outcomes were studied:

- Mode of delivery

- CTG abnormalities

- Meconium stained liquor

- Duration of labor prior to fetal compromise

- Birth weight and birth weight centiles

- Apgar score at 1 and 5 minutes

- Admission to neonatal intensive care unit (NICU)

\section{Statistical Analysis}

Data were entered in an Excel sheet and analysis was performed using SPSS.

Categorical data were presented as number and percentage (\%) and were compared using the chi-square test. Continuous data were presented as mean with SD and was compared using nonparametric tests such as the test for comparison of population means, the test for comparison of proportions, and the one-way ANOVA test. Tests of diagnostic accuracy were performed. Receiver operating characteristic (ROC) curves were used to determine the area under the curve (AUC). A $p$ value $<0.05$ was considered significant. The software used was Medcalc software version 18.5.

\section{Results}

Of the 370 women recruited, 13 were lost to follow-up and these have been excluded from the analysis. The analysis was performed on 357 subjects. Figure 1 shows the flow of participants.

Total 78 of $357(21.84 \%)$ subjects were in the CPR $<5$ th percentile group, and 279 subjects had $C P R>/=5$ th centile.

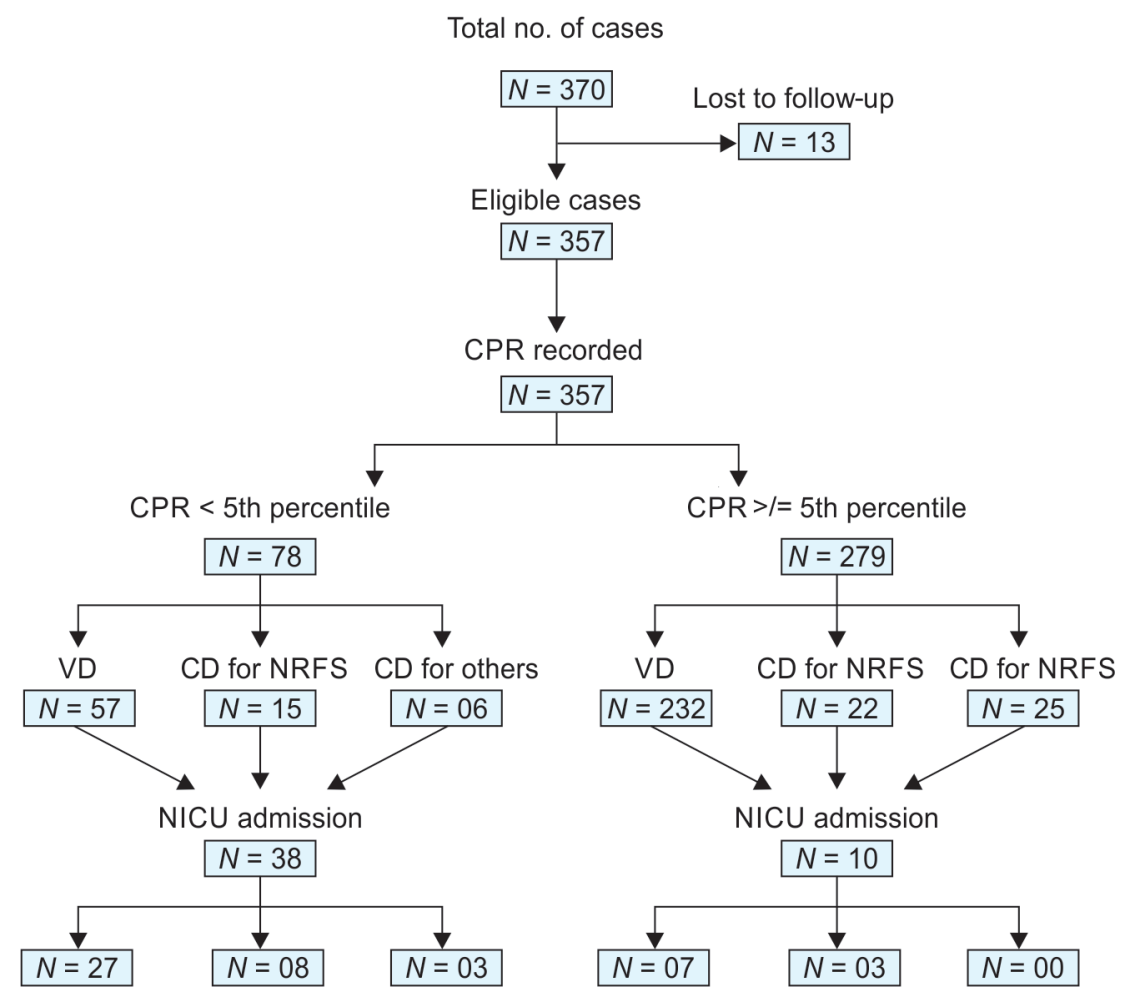

Fig. 1: Participant flow diagram 
Table 1 shows no statistical difference between two groups in terms of maternal age, parity, gestational age in days at ultrasound, gestational age in days at delivery, and interval in days between scan and delivery.

Table 2 shows that 34 of 113 (30.08\%) subjects in the $<5$ th centile group had induced labor as compared to $79(69.91 \%)$ in the $>5$ th centile group. This observation was statistically significant at $p=0.01 ; 21$ of $68(30.8 \%)$ subjects had undergone cesarean delivery of which 15 subjects required cesarean delivery for fetal distress. Difference in observations due to the mode of delivery was statistically significant at $p$ value of 0.01 .

Table 3 shows that meconium grade $2-3$ in amniotic fluid, APGAR $<7$ at 5 minutes, and number of neonates requiring admission to NICU were significantly higher in the CPR $<5$ th centile group. Birth weight centile did not affect the CPR value.

Table 4 shows the diagnostic accuracy of CPR centile for neonatal outcomes. The sensitivity for APGAR $<7$ at 5 minutes was $96 \%$ whereas it was $70 \%$ for adverse neonatal outcome in CPR $<5$ th percentile group. The positive likelihood ratio (LR) for NICU admission was 6.12, while birth weight centile had a positive LR of 1.16. The AUC for NICU admission was 0.83 . For cesarean delivery for fetal compromise, the AUC was only 0.55 (Fig. 2).

\section{Discussion}

This prospective cross-sectional study enrolled 357 subjects with low-risk singleton pregnancy at term (37-41 weeks' gestation). The CPR was evaluated with respect to maternal and fetal outcomes.
Total 78 subjects had CPR below 5th centile. The mean CPR was 1.2 at $<5$ th percentile, 1.35 at 10th percentile, and 2.56 at 90th percentile, which corresponds well with the study of Ebbing et al. ${ }^{10}$ The mean (SD) of CPR for the study population was 1.48 (+0.39); the mean (SD) value for CPR was lowest for the infants requiring NICU admission (1.12 \pm 0.22$)$.

For subjects with CPR $<5$ th centile, the odds ratio for APGAR $<7$ at 5 minutes was $8.42(1.23-57.25)$, and for NICU admission was 4.36 (1.43-13.25). These observations were similar when the analysis was performed both for the total cohort and when SGA neonates were excluded from the analysis. There were two perinatal deaths in the cohort, one each in both groups. The cause of death was necrotizing enterocolitis (NEC) and periventricular leukomalacia (PVL).

In a prospective observational study on AGA fetuses by Prior et $\mathrm{al}_{1}{ }^{1}$ the $\mathrm{C} / \mathrm{U}$ ratio at 10th percentile had value of 1.4 and the $\mathrm{C} / \mathrm{U}$ ratio at 90th percentile had value of 2.35 with mean value of 1.76 They found that a $\mathrm{C} / \mathrm{U}$ ratio $<10$ th percentile was associated with a sixfold increased rate of cesarean delivery for presumed fetal compromise when compared to $\mathrm{C} / \mathrm{U}$ ratio $>10$ th percentile. In another prospective study ${ }^{11}$ conducted by the same author, in 775 women with low-risk singleton pregnancies in early labor, fetuses with a CPR $<0.6765$ MoM significantly required cesarean delivery for fetal compromise.

Dunn et al. ${ }^{12}$ in a systematic review on utility of fetal CP ratio measured at term (37-42 weeks of gestation) found that birth by spontaneous vaginal delivery was three times more likely when CPR was normal. Whereas incidence of meconium-stained liquor was twofold greater when CPR was abnormal. This review concluded

Table 1: CPR percentile vs maternal characteristics

\begin{tabular}{|c|c|c|c|c|}
\hline Maternal characteristics & CPR percentile $<5(n=78)$ & CPR percentile $\geq 5(n=279)$ & Total population $(n=357)$ & pvalue \\
\hline \multicolumn{5}{|l|}{ Age } \\
\hline Mean & 24.65 & 24.34 & 24.41 & \multirow[t]{2}{*}{$p=0.46$} \\
\hline SD & 3.56 & 3.25 & 3.31 & \\
\hline \multicolumn{5}{|l|}{ Parity } \\
\hline Nulliparous & $40(21.73 \%)$ & $144(78.26 \%)$ & $184(51.54 \%)$ & \multirow[t]{2}{*}{$p=0.95$} \\
\hline Multiparous & $38(21.96 \%)$ & $135(78.03 \%)$ & $173(48.45 \%)$ & \\
\hline \multicolumn{5}{|c|}{ Gestational age in days at ultrasound } \\
\hline Mean & 270.93 & 270.92 & 270.93 & \multirow[t]{2}{*}{$p=0.9$} \\
\hline SD & 6.16 & 6.40 & 6.34 & \\
\hline \multicolumn{5}{|c|}{ Gestational age in days at delivery } \\
\hline Mean & 277.19 & 276.86 & 276.93 & \multirow[t]{2}{*}{$p=0.61$} \\
\hline SD & 4.76 & 5.19 & 5.09 & \\
\hline \multicolumn{5}{|c|}{ Mean interval in days between scan and delivery } \\
\hline Mean & 6.06 & 5.64 & 5.73 & \multirow[t]{2}{*}{$p=0.34$} \\
\hline SD & 3.81 & 3.40 & 3.49 & \\
\hline
\end{tabular}

Table 2: CPR percentile vs labor characteristics

\begin{tabular}{|c|c|c|c|c|}
\hline Labor characteristics & CPR percentile < $5(n=78)$ & CPR percentile $>5(n=279)$ & Total population $(n=357)$ & pvalue \\
\hline \multicolumn{5}{|l|}{ Onset of labor } \\
\hline Spontaneous & $44(18.03 \%)$ & $200(81.96 \%)$ & $244(68.34 \%)$ & $p=0.01$ \\
\hline Induced & $34(30.08 \%)$ & 79 (69.91\%) & $113(13.65 \%)$ & $x^{2}=6.56$ \\
\hline \multicolumn{5}{|l|}{ Mode of delivery } \\
\hline Vaginal delivery (VD) & $57(19.72 \%)$ & $232(80.27 \%)$ & $289(80.95 \%)$ & $p=0.014$ \\
\hline Cesarean delivery for fetal distress & $15(40.54 \%)$ & $22(59.45 \%)$ & $37(10.36 \%)$ & $X^{2}=8.44$ \\
\hline Cesarean delivery for other indication & $06(19.35 \%)$ & 25 (80.64\%) & $31(8.68 \%)$ & \\
\hline
\end{tabular}


Table 3: CPR percentile vs perinatal outcome

\begin{tabular}{|c|c|c|c|c|}
\hline Perinatal outcome & $C P R<5$ th centile $(n=33)$ & $C P R>/=5$ th centile $(n=324)$ & Total population $(n=357)$ & $p$ value \\
\hline \multicolumn{5}{|c|}{ Meconium-stained liquor } \\
\hline No meconium & $52(16.19 \%)$ & $269(83.80 \%)$ & $321(89.91 \%)$ & \multirow[t]{3}{*}{$p<0.0001$} \\
\hline Grade 2 & $16(69.56 \%)$ & 7 (30.43\%) & $23(6.44 \%)$ & \\
\hline Grade 3 & $10(76.92 \%)$ & $3(23.07 \%)$ & $13(3.64 \%)$ & \\
\hline \multicolumn{5}{|c|}{ Birth weight in grams } \\
\hline Mean & 2716.98 & 2728.92 & 2726.31 & \multirow[t]{2}{*}{$p=0.70$} \\
\hline SD & 279.26 & 253.52 & 259.0 & \\
\hline \multicolumn{5}{|c|}{ Birth weight centile in grams } \\
\hline Mean & 25.99 & 27.14 & 26.89 & \multirow[t]{2}{*}{$p=0.60$} \\
\hline SD & 23.26 & 21.61 & 21.95 & \\
\hline \multicolumn{5}{|c|}{ APGAR at 5 minutes } \\
\hline$>7$ & $49(15.36 \%)$ & $270(84.64 \%)$ & 319 (89.35\%) & \multirow[t]{2}{*}{$p<0.0001$} \\
\hline$<7$ & $29(76.31 \%)$ & $09(23.68 \%)$ & $38(10.64 \%)$ & \\
\hline \multicolumn{5}{|l|}{ NICU admission } \\
\hline Yes & $38(79.16 \%)$ & $10(20.83 \%)$ & $48(13.44 \%)$ & \multirow[t]{2}{*}{$p<0.0001$} \\
\hline No & $40(12.94 \%)$ & $269(87.05 \%)$ & 309 (86.55\%) & \\
\hline \multicolumn{5}{|c|}{ Perinatal complications } \\
\hline Yes & $17(21.79 \%)$ & $07(2.50 \%)$ & $24(6.72 \%)$ & \multirow[t]{2}{*}{$p<0.0001$} \\
\hline No & $61(78.20 \%)$ & $272(97.49 \%)$ & $333(93.27 \%)$ & \\
\hline
\end{tabular}

Table 4: Diagnostic accuracy of CPR percentile for neonatal outcomes

\begin{tabular}{llllll}
\hline $\begin{array}{l}\text { Screening efficacy of } \\
\text { CPR percentile }\end{array}$ & $\begin{array}{l}\text { APGAR<7 at } 5 \\
\text { minutes }\end{array}$ & $\begin{array}{l}\text { Meconium-stained } \\
\text { amniotic fluid }\end{array}$ & NICU admission & Mode of delivery & Birth weight centile \\
\hline Sensitivity & 96.32 & 72.2 & 79.17 & 30.88 & 24.29 \\
$(95 \%$ CI) & $(59.76,88.56)$ & $(54.81,85.8)$ & $(65.01,89.5)$ & $(20.23,43.25)$ & $(16.52,33.5)$ \\
Specificity & 84.64 & 83.8 & 87.06 & 80.27 & 79.2 \\
$(95 \%$ CI) & $(80.21,88.42)$ & $(79.3,87.66)$ & $(82.8,90.6)$ & $(75.2,84.7)$ & $(73.63,84.05)$ \\
Positive LR & 4.97 & 4.46 & 6.12 & 1.56 & 1.16 \\
$(95 \%$ centile) & $(3.6,6.8)$ & $(3.23,6.14)$ & $(4.4,8.45)$ & $(1.02,2.4)$ & $(0.77,1.76)$ \\
Negative LR & 0.28 & 0.33 & 0.24 & 0.86 & 0.95 \\
$(95 \%$ centile) & $(0.16,0.5)$ & $(0.2,0.56)$ & $(0.14,0.42)$ & $(0.72,1.01)$ & $(0.84,1.08)$ \\
Area under the curve & 0.80 & 0.78 & 0.83 & 0.55 & 051 \\
$(95 \%$ CI) & $(0.76,0.84)$ & $(0.73,0.82)$ & $(0.78,0.86)$ & $(0.5,0.6)$ & $(0.46,0.57)$ \\
\hline
\end{tabular}

that the CPR at term was strongly associated with adverse perinatal outcome.

Khalil et al. ${ }^{13}$ in a study on 7,944 pregnancies found that even after excluding the SGA fetuses, low CPR measure in multiples of median (MOM) was a predictor of adverse outcomes even in fetuses whose size is considered appropriate using conventional biometry.

Liu et al. ${ }^{14}$ in a study on 476 singleton fetuses with brain sparing within 1 week of delivery found that CPR was independently associated with the risk of categories higher categories in electronic fetal monitoring (III EFM, IIB EFM, and IIC EFM).

Bligh et al. ${ }^{15}$ in a blinded, prospective, observational, cohort study, on 483 women with uncomplicated singleton pregnancies who underwent fortnightly CPR measurement from 36 weeks to delivery, found that for cesarean section for intrapartum fetal compromise, the sensitivity was $55.5 \%(95 \% \mathrm{Cl}, 32.6-76.5)$, specificity was $87.9 \%(95 \% \mathrm{Cl}, 86.0-89.6)$, positive predictive value (PPV) was $27.0 \%(95 \% \mathrm{Cl}, 15.8-37.2)$, and negative predictive value (NPV) was $96.1 \%(95 \% \mathrm{Cl}, 94.1-97.9)$.

Sirico et al., ${ }^{16}$ in a study on 3,515 pregnancies, found that CPR MoM values are dependent on effective fetal weight (EFW) centiles. The detection rates for adverse perinatal outcomes CPR-MoM were low ranging from 6.7 to $28.6 \%$ for SGA, $12-22 \%$ for AGA, and $0-33 \%$ for LGA. Our study did not find any association between birth weight centile and $\mathrm{CP}$ ratio.

The limitations of this study are that sample size is small and the treating doctors were not blinded to the scan results. This could have led to an intervention bias. The study has not explored the effect of interobserver variability on the results.

The number of studies evaluating the role of CPR in low-risk pregnancies is small and the cut-off for abnormal CPR has not been clearly defined in this study population. There are no randomized trials that have studied the role of CPR in low-risk pregnancies at term. Most of the data come from retrospective studies.

In conclusion, this small prospective study on 357 low-risk subjects at term found that low CPR is associated with a higher risk of obstetric intervention for intrapartum fetal compromise and adverse perinatal outcomes. The CPR below the 5th centile had an independent association with Apgar $<7$ at 5 minutes, induced labor, and NICU admission. Birth weight centile did not affect this association. However, CPR in this clinical context needs to be studied in well-designed randomized clinical trials, before it can be introduced into routine clinical practice. 


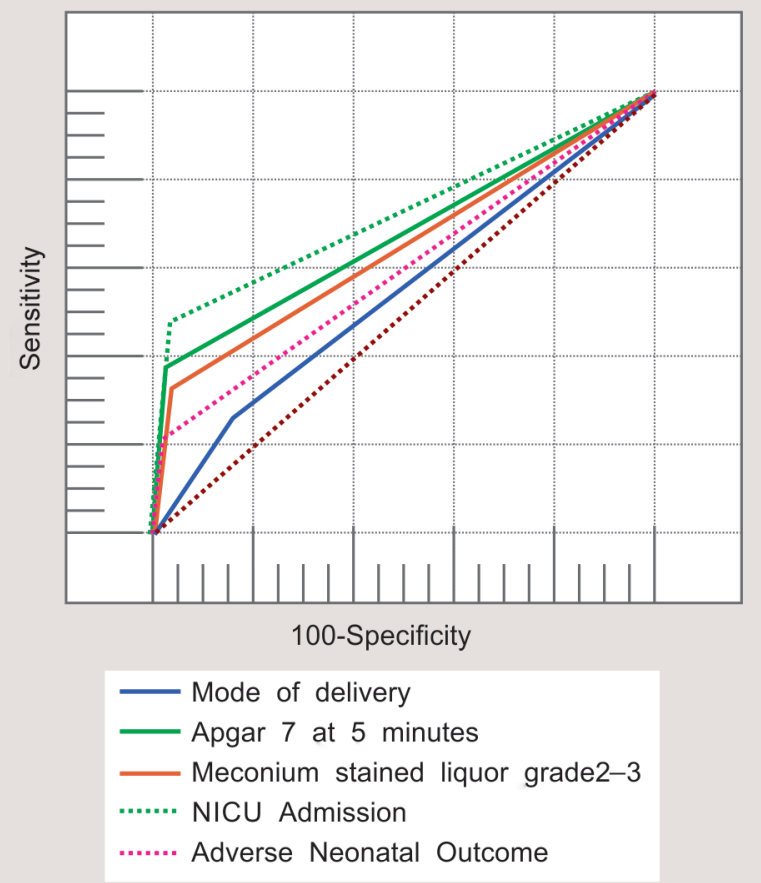

Fig. 2: Receiver operating characteristic curve for $\mathrm{CP}$ percentile and maternal and neonatal outcomes

\section{Ethics Approval}

This study was approved by the Institutional Ethics Committee: EC Reg no: ECR/85/inst/GJ/2013 dated 10/01/2017.

\section{Informed Consent}

All participants have given a written informed consent in the local language before the study began.

\section{Author Contribution}

Study concept and proposal was developed by the second author. Patient recruitment was performed by the first author. Ultrasound was performed by the second and third authors. Manuscript writing and editing was performed by all the authors.

\section{References}

1. Prior $T$, Mullins $E$, Bennett $P$, et al. Prediction of intrapartum fetal compromise using the cerebroumbilical ratio: a prospective observational study. Am J Obstet Gynecol 2013;208(2):124.e1-6. DOI: 10.1016/j.ajog.2012.11.016.
2. De Vore GR. Cerebroplacental ratio in fetal well-being in SGA and AGA fetuses. Am J Obstet Gynecol 2015;213(1):5-15. DOI: 10.1016/j. ajog.2015.05.024.

3. Flood K, Unterscheider J, Daly S, et al. The role of brain sparing in the prediction of adverse outcomes in intrauterine growth restriction: results of the multicenter PORTO study. Am J Obstet Gynecol 2014;211(3):288.e1-5. DOI: 10.1016/j.ajog.2014.05.008.

4. Morales-Roselló J, Khalil A, Morlando M, et al. Poor neonatal acid-base status in term fetuses with low cerebroplacental ratios. Ultrasound Obstet Gynecol 2015;45(2):156-161. DOI: 10.1002/uog.14647.

5. Devane D, Lalor JG, Daly S, et al. Cardiotocography versus intermittent auscultation of fetal heart on admission to labour ward for assessment of fetal wellbeing. Cochrane Database Syst Rev 2012;2(2):CD005122. DOI: 10.1002/14651858.CD005122.pub4.

6. Kushtagi P, Deepika KS. Amniotic fluid index at admission in labour as predictor of intrapartumfetal status. J Obstet Gynaecol 2011;31(5):393-395. DOI: 10.3109/01443615.2011.570811.

7. Bakalis S, Akolekar R, Gallo DM, et al. Umbilical and fetal middle cerebral artery Doppler at 30-34 weeks' gestation in the prediction of adverse perinatal outcome. Ultrasound Obstet Gynecol 2015;45(4):409-420. DOI: 10.1002/uog.14822.

8. http://medicinafetalbarcelona.org/calc/.

9. http://www.gestation.net/cc/10/624762.htm.

10. Ebbing C, Rasmussen S, Kiserud T. Middle cerebral artery blood flow velocities and pulsatility index and the cerebroplacental pulsatility ratio: longitudinal reference ranges and terms for serial measurements. Ultrasound Obstet Gynecol 2007;30(3):287-296. DOI: 10.1002/uog.4088.

11. Prior T, Paramasivam G, Bennett $P$, et al. Are babies that fail to reach their genetic growth potential at increased risk of intra-partum fetal compromise? Ultrasound Obstet Gynecol 2015;46(4):460-464. DOI: 10.1002/uog.14758.

12. Dunn L, Sherrell H, Kumar S. Review: systematic review of the utility of the fetal cerebroplacental ratio measured at term for the prediction of adverse perinatal outcome. Placenta 2017;54:68-75. DOI: 10.1016/j. placenta.2017.02.006.

13. Khalil A, Morales-Rosello J, Khan N, et al. Is cerebroplacental ratio a marker of impaired fetal growth velocity and adverse pregnancy outcome? Am J Obstet Gynecol 2017;216(6):606.e1-606.e10. DOI: 10.1016/j.ajog.2017.02.005.

14. Liu J, Song G, Zhao G, et al. The value of the cerebroplacental ratio for the prediction of intrapartum fetal monitoring in low-risk term pregnancies. Gynecol Obstet Invest 2017;82(5):475-480. DOI: 10.1159/000452664.

15. Bligh LN, Alsolai AA, Greer RM, et al. Cerebroplacental ratio thresholds measured within two weeks of birth and the risk of cesarean section for intrapartum fetal compromise and adverse neonatal outcome. Ultrasound Obstet Gynecol 2018;52(3):340-346. DOI: 10.1002/ uog. 17542 .

16. Sirico A, Diemert A, Glosemeyer P, et al. Prediction of adverse perinatal outcome by cerebroplacental ratio adjusted for estimated fetal weight. Ultrasound Obstet Gynecol 2017;51(3):381-386. DOI: 10.1002/ uog. 17458 . 